\title{
Penile Exogenous Pigmentation Mimicking Melanoma
}

\author{
Sabina Vaccari ${ }^{1}$, Michelangelo La Placa ${ }^{1}$, Alessia Barisani ${ }^{1}$, Rossella Lacava ${ }^{1}$, \\ Cosimo Misciali ${ }^{1}$, Giulio Tosti ${ }^{2}$, Valeria Gaspari ${ }^{1}$
}

\begin{abstract}
1 Dermatology - IRCCS Policlinico di Sant'Orsola, Department of Experimental, Diagnostic and Specialty Medicine (DIMES) - Alma Mater Studiorum University of Bologna, Italy

2 Division of Melanoma Surgery, Sarcoma and Rare Tumors, IRCCS, Istituto Europeo di Oncologia, Milan, Italy
\end{abstract}

Key words: penis, mucosal, exogenous, pigmentation, melanoma

Citation: Vaccari S, La Placa M, Barisani A, et al. Penile exogenous pigmentation mimicking melanoma. Dermatol Pract Concept. 2022;12(1):e2022014. DOI: https://doi.org/10.5826/dpc.1201a14

Accepted: May 6, 2021; Published: January 2022

Copyright: $@ 2022$ Vaccari et al. This is an open-access article distributed under the terms of the Creative Commons Attribution-NonCommercial License (BY-NC-4.0), https://creativecommons.org/licenses/by-nc/4.0/, which permits unrestricted noncommercial use, distribution, and reproduction in any medium, provided the original authors and source are credited.

Funding: None.

Competing interests: None.

Authorship: All authors have contributed significantly to this publication.

Corresponding author: Alessia Barisani, MD, Dermatology - IRCCS Policlinico di Sant'Orsola, Department of Experimental, Diagnostic and Specialty Medicine (DIMES) - Alma Mater Studiorum University of Bologna, Italy. Email: alessiabarisani@gmail.com

\section{Introduction}

The diagnosis of mucosal pigmented lesions can be challenging, as several differential diagnoses, including benign nevi, melanosis, melanoma, as well as post-inflammatory and physiological pigmentations, should be acknowledged. We report a case of penile exogenous pigmentation clinically and dermoscopically mimicking a malignant melanoma.

\section{Case Presentation}

A 65-year-old Caucasian man was referred to our attention because of an asymptomatic flat pigmented lesion of the penis. He reported that the lesion had appeared about 6 months earlier. Four years before, he had been diagnosed with genital lichen sclerosus and had undergone circumcision. Clinical examination revealed a brown pigmented asymmetrical flat lesion with irregular borders on the glans and the adjacent shaft (Figure 1A).
Videodermoscopy (FotoFinder dermatoscope, Fotofinder Systems, GmbH) showed a multicomponent pattern with an uneven pigmentation, multiple irregular brown-to-black dots, a blue-whitish veil and polymorphous vessels with some linear vessels at the periphery (Figure 1, B and C).

The main diagnostic suspicion was penile melanoma, due to the clinical and dermoscopic aspect and the patient's age; therefore, a skin biopsy was performed for confirmation. Histopathological examination revealed epithelium hyperplasia, sclerosis and brownish-black pigment in the superficial chorion, with no evidence of atypical melanocytic proliferation, suggestive of exogenous pigment (Figure 2, A and B). Immunohistochemistry was negative for S-100 and MART1, excluding a melanocytic neoplasm.

On the basis of the histopathological findings, a diagnosis of penile exogenous pigmentation in association with lichen sclerosus was made. We hypothesize that the lesion was a self-induced tattoo, even though the patient, suffering from a psychiatric disorder, did not confirm this hypothesis. 

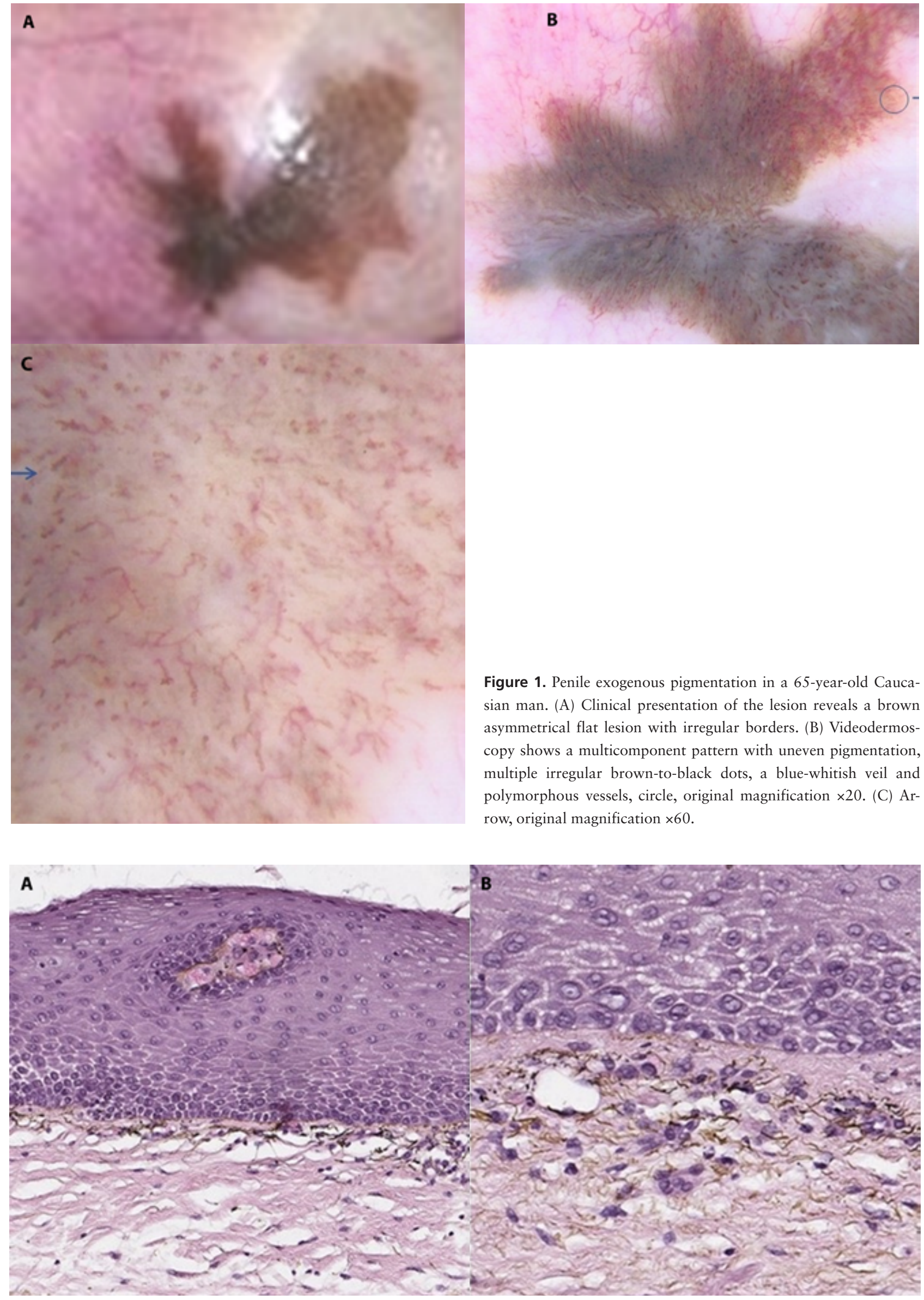

Figure 2. Penile exogenous pigmentation: histopathological findings consisting of epithelium hyperplasia, sclerosis and brownish-black pigment in the superficial chorion, with no evidence of atypical melanocytic proliferation. (A) Hematoxylin and eosin stain original magnification $\times 17$. (B) Hematoxylin and eosin stain original magnification $\times 32$. 
On the basis of the histopathological findings, a diagnosis of a penile exogenous pigmentation in association with lichen sclerosus was made.

\section{Conclusions}

Penile melanoma is a rare entity, and therefore, its dermoscopic features have been described rarely. It may show a multicomponent pattern, multiple colors (brown, black, white, blue, red), a blue-whitish veil, regression structures, and some peripheral streaks [1]. The blue, gray, and white colors and the presence of structureless areas are considered strongly suggestive of mucosal melanoma. Although videodermoscopy is a useful tool for the early diagnosis of mucosal melanoma because different colors are more easily detectable in the mucosa than in the skin, in this case it did not prove useful for the diagnosis of a mucosal exogenous pigmentation, which may show the same dermoscopic features.

The homogeneous pattern shown on dermoscopy of skin tattoos has been described rarely [2]. The peculiar dermoscopic appearance of the lesion in our patient may be due to its localization on the mucosa; and as in melanoma, dermoscopy allows a better recognition of the colors and the vascular patterns. Moreover, the associated lichen sclerosus may have altered the dermoscopic features of the lesion. Mucosal hyperpigmentation often requires the exclusion of malignant melanoma by performing histopathological examination, especially when, as in this case, the clinical history is not helpful and diagnosis is often not possible on the basis of the clinical and dermoscopic examination alone.

Informed consent: Written informed consent for publication of his clinical details and clinical images was obtained from the patient.

\section{References}

1. De Giorgi V, Grazzini M, Massi D, et al. Melanoma of the penis: a clinical dermoscopic case study. Acta Derm Venereol. 2010;90(1): 87-88. DOI: 10.2340/00015555-0705. PMID: 20107736.

2. Anthony EP, Godbolt A, Tang F, McMeniman EK. Malignant melanoma disguised in a tattoo. Australas J Dermatol. 2015;56(3):232233. DOI: 10.1111/ajd.12219. PMID: 26201372. 